\title{
Hyperuricemia- A Risk Factor of Metabolic Syndrome in Type II Diabetes with Hypertension
}

\author{
J. C. Swarnalatha', K. Vijaya Rachel², G. Amar Nagesh Kumar³, Samina Ausvi4, Anurag Yadav5
}

\begin{abstract}
${ }^{1}$ Department of Biochemistry and Bioinformatics, GITAM Deemed to be University, Rushikonda, Visakhapatnam, Andhra Pradesh, India. ${ }^{2}$ Department of Biochemistry, Institute of Sciences, GITAM University, Visakhapatnam, Andhra Pradesh, India. ${ }^{3}$ Department of Biochemistry, Karpagam Vinayaga Institute of Medical Sciences and Research Center, Madhurantagam, Tamilnadu, India. ${ }^{4}$ Department of Community Medicine, Great Eastern Medical School, Ragolu, Srikakulam, India. ${ }^{5}$ Department of Biochemistry, MNR Medical College, Fasalwadi, Sangareddy, Telangana, India.
\end{abstract}

\section{ABSTRACT}

\section{BACKGROUND}

Uric acid is the end product of purine degradation and is excreted in urine. The association between type II diabetes mellitus and Hyperuricemia has been known as early as $19^{\text {th }}$ century. Recent research in diabetic patients shows that there is an association between serum uric acid (SUA), hypertension and type II diabetes. Elevated SUA may cause increase in blood pressure by triggering inflammatory markers which in turn cause changes in the renal microvasculature, increased renin expression and endothelial dysfunction thereby resulting in hyperinsulinemia resulting in decreased disposal of glucose in type II diabetes. We wanted to compare serum uric acid level in type II diabetes and hypertension and evaluate the association of serum uric acid level with fasting blood glucose, serum creatinine and systolic blood pressure.

\section{METHODS}

This is a hospital based prospective study conducted at a tertiary care hospital. Patients were identified and recruited from the outpatient department of General Medicine. Study subjects included 137 known cases of type II diabetes mellitus and hypertension and on regular treatment for a period of more than two years. All the study subjects are in the age group of 40 to 65 years. Blood Sample collection was done in fasting condition and used for estimation of serum fasting glucose, urea, uric acid and creatinine. Anthropometric measurements were also recorded. All the results were expressed as mean and standard deviation. p Value of $<0.05$ was considered as significant. Pearson correlation was used to find out the correlation between the parameters among the study subjects.

\section{RESULTS}

In the present study, the fasting glucose, serum uric acid, urea and creatinine levels, along with the anthropometric measurements BP and BMI were estimated in type II diabetic patients diagnosed with hypertension. Results of this study demonstrated an independent correlation of serum uric acid values with serum fasting glucose levels, BP, BMI and alcoholism status, with serum urea and serum creatinine being in normal range in studied population.

\section{CONCLUSIONS}

The study provides data on cross sectional association between hyperuricemia and emphasizes the link to the future risk of metabolic syndrome (Met S) in case of type II diabetic patients with hypertension.

\section{KEY WORDS}

Type II Diabetes Mellitus, Serum Uric Acid (SUA), Hypertension, Serum Creatinine, Hyperuricemia, Metabolic Syndrome (Met S)
Corresponding Author:

Dr. K. Vijaya Rachel,

Associate Professor,

Department of Biochemistry and

Bioinformatics,

Institute of Sciences, GITAM University,

Visakhapatnam, Andhra Pradesh, India.

E-mail: gokakoushal@gmail.com

DOI: $10.14260 / \mathrm{jemds} / 2019 / 838$

Financial or Other Competing Interests: None.

How to Cite This Article:

Swarnalatha JC, Rachel KV, Kumar GAN, et al. Hyperuricemia- a risk factor of metabolic syndrome in type II diabetes with hypertension. J. Evolution Med. Dent. Sci. 2019;8(51):3868-3872,

$10.14260 /$ jemds $/ 2019 / 838$

Submission 24-10-2019,

Peer Review 06-12-2019,

Acceptance 12-12-2019,

Published 23-12-2019. 


\section{BACKGROUND}

Uric acid is the end product of purine degradation and is excreted in urine. High amounts of uric acid in blood may cause its deposition in the cooler parts of the body such as joints leading to gouty arthritis.(1) The association between type II diabetes mellitus and hyperuricemia is well recognized and has been known as early as $19^{\text {th }}$ century. Recent research in diabetic patients proves that there is an association between serum uric acid (SUA) with type II diabetes, hypertension and Metabolic syndrome.(2) SUA may cause increase in blood pressure by triggering inflammatory markers which in turn cause changes in the renal microvasculature, increased renin expression and endothelial dysfunction. $(3,4)$

These effects of elevated uric acid are caused by the injury to the endothelial cells and smooth muscle, by initiating the conversion of xanthine dehydrogenase to xanthine oxidase.(5) Furthermore, there are hypotheses, which state that fructose promotes the production of uric acid very rapidly promoting type II diabetes combined with insulin resistance.(6) Elevated SUA has also been associated with metabolic syndrome, type II diabetes and other cardiovascular risk factors. (7,8) Other researchers have observed that uric acid is a potential independent confounder of cardiovascular mortality.(9)

It is also identified that depletion of intra cellular ATP and elevated serum uric acid may have important roles in the ability of fructose to induce features of type II diabetes. The renal output of uric acid is decreased on the proximal tubule of the kidney leading to hyperuricemia, causing reduced NO availability, thereby resulting in hyperinsulinemia.(10) Hyperinsulinemia, influences renal tubular function that causes decreased urinary uric acid clearance with decreasing insulin mediated glucose disposal.(11)

SUA levels in adult patients with type II diabetes tend to be higher than in the general population, and are strongly related to development of diabetic kidney disease.(12) Elevated serum uric acid levels are associated with insulin resistant syndrome and are also associated with the early onset or increased progression of nephropathy.(13) Previous studies have identified that the incidence of hyperuricemia and gout symptoms were mainly prominent in pre-diabetes and that the risk was lower at highly elevated HbA1c. Increased xanthine oxidase activity results in the production of uric acid from purine precursors, with the resultant generation of free radicals, there may be injury to the artery.(14)

The elevated SUA levels in patients with type II diabetes may also change its nature by changing age.(15) Therefore, there is a need to better understand the association between SUA and BP in adults with and without type II diabetes. The present study aims to find out the association of serum uric acid with fasting serum glucose, urea, creatinine and blood pressure in type II diabetic patients with hypertension.

\section{METHODS}

The study is a hospital based prospective study conducted at GEMS Medical College and Hospital, Srikakulam, Andhra Pradesh after obtaining an ethical clearance certificate from the ethical board. Patients are identified and recruited from the outpatient department of General Medicine, GEMS Medical
College and Hospital, Srikakulam, Andhra Pradesh. Study subjects include 137 patients who are already diagnosed with type II diabetes mellitus and hypertension and on regular treatment for a period of more than two years. Sample size was taken based on the convenience of the study. They were being ordered for serum uric acid first time. Patients who are already diagnosed with Gout and on treatment with allopurinol or inhibitors of xanthine oxidase, patients with Chronic Kidney diseases, hyper or hypothyroidism, smokers and patients who are all on treatment with drugs that cause decreased excretion of uric acid like, pyrazinamide, ethambutol, nicotinic acid, cyclosporin, 2-ethylamino-1, 3, 4-thiadiazole and cytotoxic agents were excluded from the study. Blood pressure was checked using sphygmomanometer and readings are noted. Medical history of subjects along with investigations and treatment has been collected in a questionnaire format with their consent.

Blood collection was done in fasting condition from median cubital vein puncture and blood is distributed in plain and fluoride tubes as per the requirement. To separate serum, centrifugation of samples is carried out at a speed of $5000 \mathrm{rpm}$ using Remi centrifuge for 10 minutes. After centrifugation, blood samples were estimated for fasting glucose, urea, uric acid and creatinine-

- Serum Glucose is estimated by Hexokinase Method.

- Serum Urea is estimated by Urease method,

- Serum Uric acid is estimated by Uricase enzymatic method and

- Serum Creatinine is estimated by Jaffe's method on Biochemistry Analyzer (Roche Cobas c311)

Anthropometric measurements of all the participant patients were recorded. Blood Pressure (BP) was recorded, Body Mass Index (BMI) of all participant patients was calculated using their height and weight measurements.

\section{Statistical Analysis}

All the results were expressed as mean and standard deviation. ${ }^{*} p$ value of $<0.05$ was considered as significant. Pearson correlation was used to find out the association between the parameters among the study subjects. Statistical analysis was done by SPSS software version 16.0.

\section{RESULTS}

A total of 137 patients were consented into the study. All the study subjects are in the age group of 40 to 65 years. Out of 137 patients, 74 were male patients and 63 were female patients. The mean age of the patients was 53 years. All the patients are diabetic and hypertensive and were on mixed diet. Normal range of fasting glucose is $70-110 \mathrm{mg} / \mathrm{dl}$, urea is $15-$ $40 \mathrm{mg} / \mathrm{dl}$, creatinine is $0.6-1.3 \mathrm{mg} / \mathrm{dl}$ and uric acid is 3.5 to 6.5 $\mathrm{mg} / \mathrm{dl}$ respectively.

The mean values in the studied population are as follows, fasting sugar $134.5 \pm 48.8 \mathrm{mg} / \mathrm{dl}$, urea $25.9 \pm 8.5 \mathrm{mg} / \mathrm{dl}$, creatinine $1.35 \pm 0.31 \mathrm{mg} / \mathrm{dl}$ and uric acid $6.68 \pm 2.5 \mathrm{mg} / \mathrm{dl}$ (Table 1). An average of 3 readings of BP was taken. Mean systolic blood pressure is $137.9 \pm 13.9$ while the diastolic blood pressure is $84.1 \pm 7.7 \mathrm{mmHg}$ (Table 1). The average BMI calculated was 28.16. BMI, alcoholic status, fasting sugar, BP 
and serum uric acid were used as the markers of metabolic syndrome. The questionnaire format included the information about the nature of diet, occupation, health condition, medication and alcoholism. No patient was a smoker.

\begin{tabular}{|c|c|c|}
\hline Parameter & Mean & Standard Deviation \\
\hline Age (Years) & 53.78 & 11.08 \\
\hline Fasting Glucose (mg/dl) & 134.59 & 48.86 \\
\hline Urea (mg/dl) & 25.93 & 8.58 \\
\hline Uric acid (mg/dl) & 6.68 & 1.51 \\
\hline Creatinine (mg/dl) & 1.35 & 0.31 \\
\hline Systolic B.P. (mm of Hg) & 137.94 & 13.97 \\
\hline Diastolic B.P. (mm of Hg) & 84.15 & 7.78 \\
\hline BMI & 28.16 & 2.12 \\
\hline Table 1. Baseline Characteristics of Studied Parameters in \\
Type II Diabetic Patients \\
\hline
\end{tabular}

\begin{tabular}{|c|c|c|c|c|c|c|c|}
\hline & 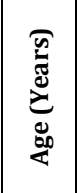 & 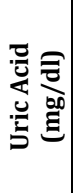 & 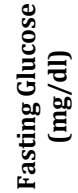 & 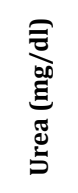 & 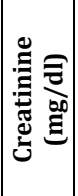 & 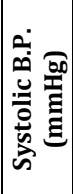 &  \\
\hline Age (years) & 1 & - & - & - & - & - & - \\
\hline Uric Acid (mg/dl) & 0.164 & 1 & - & - & - & - & - \\
\hline \begin{tabular}{|l} 
Fasting Glucose $(\mathrm{mg} / \mathrm{dl})$ \\
\end{tabular} & -0.052 & 0.467 & 1 & - & - & - & - \\
\hline Urea $(\mathrm{mg} / \mathrm{dl})$ & 0.240 & 0.030 & 0.055 & 1 & - & - & - \\
\hline Creatinine $(\mathrm{mg} / \mathrm{dl})$ & 0.167 & 0.589 & -0.091 & 0.159 & 1 & - & \\
\hline Systolic B.P. (mmHg) & 0.054 & 0.627 & -0.075 & -0.010 & 0.414 & 1 & - \\
\hline Diastolic B.P. (mmHg) & -0.055 & 0.251 & -0.005 & -0.060 & 0.169 & 0.544 & 1 \\
\hline
\end{tabular}


Table 2 shows the Pearson correlation among the studied parameters. From Table 2, it is evident that serum uric acid shows a strong positive correlation with serum creatinine $(\mathrm{r}=0.58916 ; \mathrm{p}<0.05)$, systolic blood pressure $(\mathrm{r}=0.62702 ; \mathrm{p}$ $<0.05$ ) and with fasting serum glucose ( $r=0.467, \mathrm{p}<0.05)$. Similarly, serum creatinine also shows positive correlation with systolic blood pressure in type II diabetic patients with hypertension. The average systolic pressure of the hyperuricaemic males was $200 \mathrm{mmHg}$ and that of the average systolic pressure of hyperuricaemic females was $180 \mathrm{mmHg}$. (Fig 1a).Out of the 63 female patients, 27 patients showed an elevated uric acid whose mean was about $7.16 \mathrm{mg} / \mathrm{dl}$. The average mean of serum uric acid of the 17 hyperuricaemic males was $7.82 \mathrm{mg} / \mathrm{dl}$. (Fig 1b).

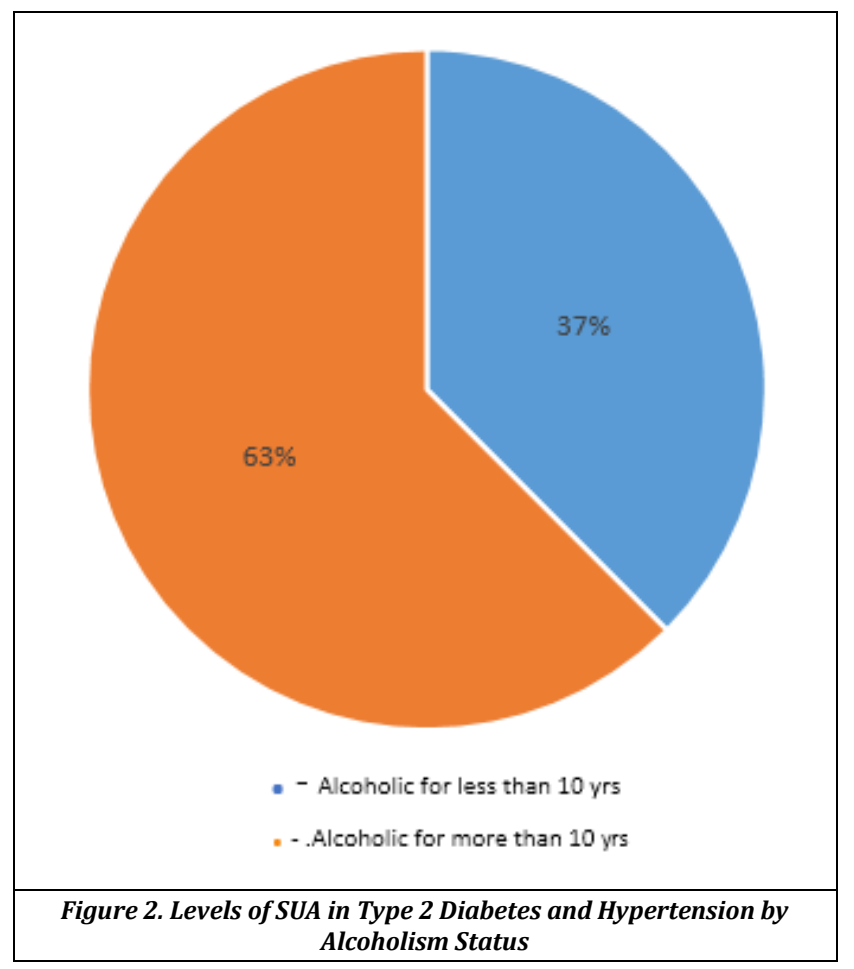

\section{DISCUSSION}

Researches are being carried out to explain the underlying mechanisms between the association of hyperuricemia and type II diabetes with hypertension. Here, we attempted to discuss the same aspect by estimating the fasting blood glucose, creatinine and systolic BP with respect to elevated serum uric acid in the patients suffering from type II diabetes and hypertension for a period of over 5 years.

Our results showed a positive correlation between serum uric acid and fasting blood sugar which are in line with the previous studies.(16) Similar studies have tried to explain that men with high fasting plasma have high risk of developing impaired glucose tolerance in association with hyperuricemia.(17) Thus, although our study provides support for the independent association between SUA levels and the risk of incident type II diabetes, any causal interference remains to be clarified in future studies.

Insulin resistance may be the linking between elevated glucose and uric acid levels.(18) Preliminary investigations also suggest that there is an indication of hyperuricemia is indeed a component of Metabolic Syndrome and could also be used as a simple marker of insulin resistance. This link was explained by two mechanisms 1) reduction of NO bioavailability and endothelial supply, which inhibits delivery of glucose to the skeletal muscle(19) and 2) activation of NADPH oxidase, generating oxidized lipids and inflammatory mediators in adipocytes.(20) Our study also demonstrated the increase in 
serum uric acid levels in type II diabetes, which corresponds to the studies on higher risk for developing impaired glucose tolerance and type II diabetes. (21) However, there are studies with negative association of uric acid with overt diabetes, ${ }^{(22,23)}$ therefore, there is a need to better understand the underlying mechanisms that explain the link between high uric acid and impaired glucose tolerance.

Serum creatinine serves as a sensitive indicator in the early diagnosis of kidney failure, so we tried estimating the serum creatinine in these patients which showed a positive correlation with serum uric acid. The mechanism of which has been explained as hyperuricemia leads to incident kidney disease.(24) It is said that uric acid mediates the relation between hypertension and renal disease via renal vasoconstriction and systemic hypertension. A common hypothesis also exists that the common association of hyperuricemia with CKD was solely attributed to the retention of serum uric acid that is known to occur as the glomerular filtration rate falls.(25) This signifies the estimation of creatinine and Microalbumin in the urine samples of these patients as there is a general consensus that evaluation of Microalbuminuria is useful for the assessment of overall cardiovascular risk in hypertension. This appears to be an advantageous method to identify patients at higher risk for whom additional preventive and therapeutic measures are advisable.(26) The link between uric acid and arterial HTN was first noted in the 1960s. As observed in the existing research, there are two mechanisms involved, one being mediated by direct entry of uric acid into both endothelial and vascular smooth muscle cells, resulting in local inhibition of endothelial nitric oxide levels, stimulation of vasoactive and inflammatory mediators.(27)

Uric acid and BMI were positively correlated in our study. The individuals who were suffering from only diabetes showed a strong correlation than the hypertensive and patients suffering both hypertension and diabetes. The prevalence of hyperuricemia in our study was 2 times more in obese patients when compared to normal weight diabetics. This shows that obesity may serve as a clinical indicator for hyperuricemia. Though the mechanism pertaining to the association between elevated BMI and elevated serum uric acid is not well established, the possible reason has been observed as the abnormal fat distribution in the body.(28) Uric acid and consumption of alcohol, we studied a combination of patient population which included alcoholic and non-alcoholic subjects. When we estimated serum uric acid in both hypertensives and diabetics, subjects who were alcoholic for more than 10 years with continuous intake of alcohol showed a 2 -fold rise in their serum uric acid value than the normal range (Fig. 2). The possible reason behind this elevation might be due to the over production of lactate due to alcoholic intake.(29)

\section{CONCLUSIONS}

In this study, a markedly elevated serum uric acid was seen in patients with type II diabetes suffering from hypertension. This is in-line with some previous studies, which suggest a possible link between uric acid levels and type II diabetes and hypertension. Interestingly, serum uric acid levels were increased in type 2 diabetic patients who have a history of hypertension and this phenomenon seemed to be more profound in male diabetic patients, who also demonstrated more prominent effect of glucose control on UA level than their female counterparts. Since, to our knowledge, this is one of the first studies addressing prevalence of hyperuricemia in Andhra Pradesh and its role in diabetes progression, more emphasis should be put on this risk factor for developing metabolic and cardiovascular disease in clinical practice in our country. Additional genetic studies addressing gender and ethno specific effects on UA levels in type 2 diabetic patients also appear to be justified. Thus, considering the potential link of elevated serum uric acid with insulin resistance, impaired glucose tolerance, and progression to diabetes, further research should be attempted to determine as to whether it is effective to utilize fasting glucose, serum creatinine and uric acid levels as a predictor in prevention of Type II diabetes associated hypertension included metabolic syndrome. However, our study falls short in not taking the total cholesterol value of the study population into consideration and the sample size is also limited.

\section{ACKNOWLEDGEMENT}

Authors thank the Management and Principal of GEMS Medical College and Hospital, Srikakulam, Andhra Pradesh for allowing them to conduct this research study. Authors also would like to thank GITAM Deemed to be University, Visakhapatnam, for its support.

\section{REFERENCES}

[1] Kumar AN, Kumar R, Aruna P, et al. Review of concepts and controversies of uric acid as antioxidant and prooxidant. Archives Medical Review Journal 2015;24(1):1940.

[2] Bhole V, Choi JW, Kim SW, et al. Serum uric acid levels and the risk of type ii diabetes: a prospective study. Am J Med 2010;123(10):957-61.

[3] Buscemi S, Nicolucci A, Lucisano G, et al. Impact of chronic diuretic treatment on glucose homeostasis. Diabetol Metab Syndr 2013;5(1):80.

[4] Choi HK, Ford ES. Prevalence of the metabolic syndrome in individuals with hyperuricemia. American Journal of Medicine 2007;120(5):442-7.

[5] Jin M, Yang F, Yang I, et al. Uric acid, hyperuricemia and vascular diseases. Front Biosci (Landmark Ed) 2012;17:656-69.

[6] Choi HK, Ford ES. Haemoglobin A1c, fasting glucose, serum C-Peptide and insulin resistance in relation to erum uric acid levels. The Third National Health and Nutrition Examination Survey. Rheumatology (Oxford) 2008;47(5):713-7.

[7] Nakagawa T, Tuttle KR, Short RA, et al. Hypothesis: fructose-induced hyperuricemia as a causal mechanism for the epidemic of the metabolic syndrome. Nature Clinical Practice Nephrology 2005;1(2):80-6. 
[8] Genuth S, Alberti KG, Bennett P, et al. Follow-up report on the diagnosis of diabetes mellitus. Diabetes Care 2003;26(11):3160-7.

[9] Andrikou I, Tsioufis C, Dimitriadis K, et al. Uric acid as an independent predictor of coronary artery disease in essential hypertension: Data from an 8-year-follow-up study. Clinical and Experimental Pharmocology and Physiology, Letter to the editor 2018;45(8):866-9.

[10] Li C, Hsieh MC, Chang SJ. Metabolic syndrome, diabetes and hyperuricemia. Curr Opin Rheumatol 2013;25(2):210-6.

[11] Zoppini G, Targher G, Negri C, et al. Elevated serum uric acid concentrations independently predict cardiovascular mortality in Type II diabetic patients. Diabetes Care 2009;32(9):1716-20.

[12] Quinones-Galvan A, Ferrannini E. Renal effects of insulin in man. J Nephrol 1997;10(4):188-91.

[13] Bo S, Cavallo-Perin P, Gentile L, et al. Hypouricemia and Hyperuricemia in type II diabetes: two different phenotypes. Eur J Clin Invest 2001;31(4):318-21.

[14] Dawson J, Walters M. Uric acid and xanthine oxidase: future theraupeutic targets in the prevention of cardiovascular disease? $\mathrm{Br}$ J Clin Pharmacol 2006;62(6):633-44.

[15] Lee JJ, Ahn J, Hwang J, et al. Relationship between uric acid and blood pressure in different age groups. Clin Hyperten 2015;21:14.

[16] Kodama S, Saito K, Yachi Y, et al. Association between serum Uric acid and development of type 2 Diabetes. Diabetes Care 2009;32(9):1737-42.

[17] Miyake T, Kumagi T, Furukawa S, et al. Hyperuricemia is a risk factor for the onset of impaired fasting glucose in men with a high plasma glucose level: a community based study PLoS One 2014;9(9): e0107882.

[18] Modan M, Halkin H, Karasik A, et al. Elevated serum uric acid - a facet of hyperinsulinemia. Diabetologia 1987;30(9):713-8.
[19] Vuorinen-Markkola H, Yki-Jarvinen H. Hyperuricemia and insulin resistance. J Clin Endocrinol Metab 1994;78(1):25-9.

[20] Sautin YY, Nakagawa T, Zharikov S, et al. Adverse effects of the classic antioxidant uric acid in adipocytes: NADPH oxidase- mediated oxidative/nitrosative stress. Am J Physiol Cell Physiol 2007;293(2):C584-96.

[21] Meisinger C, Doring A, Stockl D, et al. Uric acid is more strongly associated with impaired glucose regulation in women than in men from the general population: The KORA F4-Study. PLoS One 2012;7(5): e37180.

[22] Bandaru P, Shankar A. Association between serum uric acid levels and diabetes mellitus. International Journal of Endocrinology 2011;2011:604715.

[23] Causevic A, Semiz S, Macic-Dzankovic A, et al. Relevance of uric acid in progression of type II Diabetes mellitus. Bosnian Journal of Basic Sciences 2010;10(1):54-9.

[24] Weiner DE, Tighiouart H, Elsayed EF, et al. Uric acid and incident kidney disease in the community. J Am Soc Nephrol 2008;19(6):1204-11.

[25] Johnson RJ, Segal MS, Srinivas T, et al. Essential hypertension, progressive renal disease and uric acid: a pathogenetic link? J Am Soc Nephrol 2005;16(7):1909-19.

[26] Palatini P. Microalbuminuria in hypertension. Curr Hypertens Rep 2003;5(3):208-14.

[27] Johnson RJ, Nakagawa T, Jalal D, et al. Uric acid and chronic kidney disease: which is chasing which? Nephrol Dial Transplant 2013;28(9):2221-8.

[28] Matsuura F, Yamashita S, Nakamura T, et al. Effect of visceral fat accumulation on uric acid metabolism in male obese subjects: visceral fat is linked more closely to overproduction of uric acid than sub cutaneous fat obesity. Metabolism 1998;47(8):929-33.

[29] Lieber CS. Hyperuricemia induced by alcohol. Arthritis and Rheumatism 1965;8(5):786-98. 